\title{
CARTHAGINIAN ENLARGEMENT OF FILTRATIONS *
}

\author{
Giorgia Callegaro $^{1,2}$, Monique Jeanblanc ${ }^{3}$ and Behnaz Zargari ${ }^{4}$
}

\begin{abstract}
This work is concerned with the theory of initial and progressive enlargements of a reference filtration $\mathbb{F}$ with a random time $\tau$. We provide, under an equivalence assumption, slightly stronger than the absolute continuity assumption of Jacod, alternative proofs to results concerning canonical decomposition of an $\mathbb{F}$-martingale in the enlarged filtrations. Also, we address martingales' characterization in the enlarged filtrations in terms of martingales in the reference filtration, as well as predictable representation theorems in the enlarged filtrations.
\end{abstract}

Mathematics Subject Classification. 60G46, 60-02.

Received January 24, 2011. Revised September 28, 2011.

\section{INTRODUCTION}

We consider the case where a filtration $\mathbb{F}$ is enlarged to give a filtration $\widetilde{\mathbb{F}}$, by means of a finite positive random variable $\tau$. In the literature, two ways to realize such an enlargement are presented: either all of a sudden at time 0 (initial enlargement), or progressively, by considering the smallest filtration containing $\mathbb{F}$, satisfying the usual conditions, that makes $\tau$ a stopping time (progressive enlargement).

The "pioneers" who started exploring this research field, at the end of the seventies, were Barlow (see [4]), Jacod et al. (see the references that follow in the text). The main questions that raised were the following: "does any $\mathbb{F}$-martingale $X$ remain an $\widetilde{\mathbb{F}}$-semimartingale?" And, if it does: "what is the semimartingale decomposition in $\widetilde{\mathbb{F}}$ of the $\mathbb{F}$-martingale $X$ ?"

The main contribution of the present work is to show how, under a specific equivalence assumption (see Assumption 2.1), slightly stronger than Jacod's one in [15], some well-known fundamental results can be proved in an alternative (and, in some cases, simpler) way. We make precise that the goal of this paper is neither to present the results in the most general case, nor to study the needed and difficult regularity properties, for which we refer to existing papers (e.g., Jacod [15]).

Keywords and phrases. Initial and progressive enlargements of filtrations, predictable projection, canonical decomposition of semimartingales, predictable representation theorem.

* This research was supported by the "Chaire Risque de Crédit" of the French Banking Federation. All the authors are members of the Université d'Evry Val d'Essonne, Laboratoire d'Analyse et Probabilités, 23 Boulevard de France, 91037 Evry cedex, France.

1 Scuola Normale Superiore di Pisa, Piazza dei Cavalieri, 756126 Pisa, Italy. giogiocallegaro@gmail.com

2 CREST, 15 Boulevard Gabriel Péri, 92254 Malakoff Cedex, France.

3 Institut Europlace de Finance (EIF), Palais Brongniart, 28 Place de la Bourse, 75002 Paris, France.

monique.jeanblanc@univ-evry.fr

4 Sharif University of Technology, P.O. Box 11365-8639, Tehran, Iran. behnaz_zargari@yahoo.com 
Let us, now, motivate the title. Inspired by a visit to the Tunisian archaeological site of Carthage, where one can find remains of THREE levels of different civilizations, we decided to use the catchy adjective "Carthaginian" associated with filtration, since in this paper there will be THREE levels of filtrations.

We consider, then, three nested filtrations

$$
\mathbb{F} \subset \mathbb{G} \subset \mathbb{G}^{\tau}
$$

where $\mathbb{G}$ and $\mathbb{G}^{\tau}$ stand, respectively, for the progressive and the initial enlargement of $\mathbb{F}$ with a finite random time $\tau$ (i.e., a finite non-negative random variable).

Under a specific assumption (see the $(\mathcal{E})$-hypothesis below), we address the following problems:

- characterization of $\mathbb{G}$-martingales and $\mathbb{G}^{\tau}$-martingales in terms of $\mathbb{F}$-martingales;

- canonical decomposition of an $\mathbb{F}$-martingale, as a semimartingale, in $\mathbb{G}$ and $\mathbb{G}^{\tau}$;

- predictable representation theorem in $\mathbb{G}$ and $\mathbb{G}^{\tau}$.

The exploited idea is the following: assuming that the $\mathbb{F}$-conditional law of $\tau$ is equivalent to the law of $\tau$, after an ad hoc change of probability measure, the problem reduces to the case where $\tau$ and $\mathbb{F}$ are independent. Under this newly introduced probability measure, working in the initially enlarged filtration is "easy". Then, under the original probability measure, for the initially enlarged filtration, the results are achieved by means of Girsanov's theorem. As for the progressively enlarged filtration, one can proceed either by projecting on $\mathbb{G}$ the results already obtained for $\mathbb{G}^{\tau}$ (e.g., in Prop. 3.3), or, directly, by a change of probability measure in the filtration $\mathbb{G}$ (e.g., in Prop. 5.5 (ii)).

The "change of probability measure" viewpoint for treating problems on enlargement of filtrations was remarked in the early 80's and developed by Song in [22], and then by Ankirchner et al. [2] (see also [15], Sect. 5). This is also the point of view adopted by Gasbarra et al. in [11] where the authors apply the Bayesian approach to study the impact of the initial enlargement of filtration on the characteristic triplet of a semimartingale.

The paper is organized as follows. Section 2 introduces definitions and preliminary results which will be crucial in the rest of the paper. Section 3 addresses the characterization of $\mathbb{G}$-martingales and $\mathbb{G}^{\tau}$-martingales in terms of $\mathbb{F}$-martingales. In Section 4 , the invariance of semimartingale property under the progressive and initial enlargements of filtration is studied, and the formulae for the canonical decomposition of an $\mathbb{F}$-martingale as a semimartingale in $\mathbb{G}$ and $\mathbb{G}^{\tau}$ are provided. In Section 5, we show that the enlarged filtrations $\mathbb{G}$ and $\mathbb{G}^{\tau}$ admit a predictable representation property, as soon as the reference filtration $\mathbb{F}$ enjoys one. Finally, Section 6 ends the paper with some concluding remarks.

\section{Preliminaries}

We consider a probability space $(\Omega, \mathcal{A}, \mathbb{P})$ equipped with a filtration $\mathbb{F}=\left(\mathcal{F}_{t}\right)_{t>0}$ satisfying the usual hypotheses of right-continuity and completeness, and where $\mathcal{F}_{0}$ is the trivial $\sigma$-field, completed by the $\mathbb{P}$-negligible sets of $\mathcal{A}$.

Let $\tau$ be a finite random time with law $\nu, \nu(\mathrm{d} u)=\mathbb{P}(\tau \in \mathrm{d} u)$. We assume that $\nu$ has no atoms and has $\mathbb{R}^{+}$ as support.

We denote by $\mathcal{P}(\mathbb{F})$ (resp. $\mathcal{O}(\mathbb{F})$ ) the predictable (resp. optional) $\sigma$-algebra corresponding to $\mathbb{F}$ on $\mathbb{R}^{+} \times \Omega$.

Our standing assumption is the following:

Assumption $2.1((\mathcal{E})$-hypothesis). The $\mathbb{F}$-(regular) conditional law of $\tau$ is equivalent to the law of $\tau$. Namely,

$$
\mathbb{P}\left(\tau \in \mathrm{d} u \mid \mathcal{F}_{t}\right) \sim \nu(\mathrm{d} u) \text { for every } t \geq 0, \mathbb{P}-\text { a.s. }
$$

This assumption, in the case where $t \in[0, T]$, corresponds to the equivalence assumption in Föllmer and Imkeller [10] and in Amendinger's thesis [1], Assumption 0.2, and to hypothesis (HJ) in the papers by Grorud and Pontier (see, e.g., [12]). 
Amongst the consequences of the $(\mathcal{E})$-hypothesis, one has the existence and regularity of the conditional density, for which we refer to Amendiger's reformulation (see remarks in p. 17 of [1]) of Jacod's result (Lem. 1.8 in [15]): there exists a strictly positive $\mathcal{O}(\mathbb{F}) \otimes \mathcal{B}\left(\mathbb{R}^{+}\right)$-measurable function $(t, \omega, u) \rightarrow p_{t}(\omega, u)$, such that for $\nu$-almost every $u \in \mathbb{R}^{+}, p(u)$ is a càdlàg $(\mathbb{P}, \mathbb{F})$-martingale and

$$
\mathbb{P}\left(\tau>\theta \mid \mathcal{F}_{t}\right)=\int_{\theta}^{\infty} p_{t}(u) \nu(\mathrm{d} u) \quad \text { for every } t \geq 0, \quad \mathbb{P}-\text { a.s. }
$$

In particular, $p_{0}(u)=1$ for $\nu$-almost every $u \in \mathbb{R}^{+}$. This family of processes $p$ is called the $(\mathbb{P}, \mathbb{F})$-conditional density of $\tau$ with respect to $\nu$, or the density of $\tau$ if there is no ambiguity.

Furthermore, under the $(\mathcal{E})$-hypothesis, the assumption that $\nu$ has no atoms implies that the default time $\tau$ avoids the $\mathbb{F}$-stopping times, i.e., $\mathbb{P}(\tau=\xi)=0$ for every $\mathbb{F}$-stopping time $\xi$ (see, e.g., Cor. 2.2 in El Karoui et al. $[9])$.

The initial enlargement of $\mathbb{F}$ with $\tau$, denoted by $\mathbb{G}^{\tau}=\left(\mathcal{G}_{t}^{\tau}, t \geq 0\right)$, is defined as $\mathcal{G}_{t}^{\tau}=\mathcal{F}_{t} \vee \sigma(\tau)$. It was shown in [1], Proposition 1.10, that the strict positiveness of $p$ implies the right-continuity of the filtration $\mathbb{G}^{\tau}$.

Let $\mathbb{H}=\left(\mathcal{H}_{t}\right)_{t>0}$ denote the smallest filtration with respect to which $\tau$ is a stopping time, i.e., $\mathcal{H}_{t}=$ $\sigma\left(\mathbb{1}_{\tau \leq s}, s \leq t\right)$. This filtration is right-continuous. The progressive enlargement of $\mathbb{F}$ with the random time $\tau$, denoted by $\mathbb{G}=\left(\mathcal{G}_{t}\right)_{t>0}$, is defined as the right-continuous regularization of $\mathbb{F} \vee \mathbb{H}$.

In the sequel, we will consider the right-continuous version of all the martingales.

Now, we consider the change of probability measure introduced, independently, by Grorud and Pontier in [12] and by Amendinger in [1]. Having verified that the process $L$, given by $L_{t}=\frac{1}{p_{t}(\tau)}, t \geq 0$, is a $\left(\mathbb{P}, \mathbb{G}^{\tau}\right)$-martingale, with $\mathbb{E}\left(L_{t}\right)=L_{0}=1$, these authors defined a locally equivalent probability measure $\mathbb{P}^{*}$ setting

$$
\mathrm{dP}^{*}{ }_{\mid \mathcal{G}_{t}^{\tau}}=L_{t} \mathrm{~d} \mathbb{P}_{\mid \mathcal{G}_{t}^{\tau}}=\frac{1}{p_{t}(\tau)} \mathrm{d} \mathbb{P}_{\mid \mathcal{G}_{t}^{\tau}} .
$$

They proved that, under $\mathbb{P}^{*}$, the random time $\tau$ is independent of $\mathcal{F}_{t}$ for any $t \geq 0$ and, moreover, that

$$
\mathbb{P}^{*} \mathcal{F}_{t}=\mathbb{P}_{\mid \mathcal{F}_{t}} \text { for any } t \geq 0, \quad \mathbb{P}^{*}{ }_{\mid \sigma(\tau)}=\mathbb{P}_{\mid \sigma(\tau)} .
$$

The above properties imply that $\mathbb{P}^{*}\left(\tau \in \mathrm{d} u \mid \mathcal{F}_{t}\right)=\mathbb{P}^{*}(\tau \in \mathrm{d} u)$, so that the $\left(\mathbb{P}^{*}, \mathbb{F}\right)$-density of $\tau$, denoted by $p^{*}(u), u \geq 0$, is a constant equal to one, $\mathbb{P}^{*} \otimes \nu$-a.s.

Remark 2.2. The probability measure $\mathbb{P}^{*}$, being defined on $\mathcal{F}_{t}$ for $t \geq 0$, is (uniquely) defined on $\mathcal{F}_{\infty}=\bigvee_{t \geq 0} \mathcal{F}_{t}$. Then, as $\tau$ is independent of $\mathbb{F}$ under $\mathbb{P}^{*}$, it immediately follows that $\tau$ is also independent of $\mathcal{F}_{\infty}$, under $\mathbb{P}^{*}$. However, one can not claim that: " $\mathbb{P}^{*}$ is equivalent to $\mathbb{P}$ on $\mathcal{G}_{\infty}^{\tau}$ ", since we do not know a priori whether $\frac{1}{p(\tau)}$ is a closed $\left(\mathbb{P}, \mathbb{G}^{\tau}\right)$-martingale or not. A similar problem is studied by Föllmer and Imkeller in [10] (it is therein called "paradox") in the case where the reference (canonical) filtration is enlarged by means of the information about the endpoint at time $t=1$. In our setting, it corresponds to the case where $\tau \in \mathcal{F}_{\infty}$ and $\tau \notin \mathcal{F}_{t}, \forall t$.

Remark 2.3. Let $x=\left(x_{t}, t \geq 0\right)$ be a $(\mathbb{P}, \mathbb{F})$-local martingale. Since $\mathbb{P}$ and $\mathbb{P}^{*}$ coincide on $\mathbb{F}, x$ is a $\left(\mathbb{P}^{*}, \mathbb{F}\right)$-local martingale, hence, using the fact that $\tau$ is independent of $\mathbb{F}$ under $\mathbb{P}^{*}$, a $\left(\mathbb{P}^{*}, \mathbb{G}\right.$ )-local martingale (and also a $\left(\mathbb{P}^{*}, \mathbb{G}^{\tau}\right)$-local martingale).

Notation 2.4. In this paper, as we mentioned, we deal with three different levels of information and two equivalent probability measures. In order to distinguish objects defined under $\mathbb{P}$ and under $\mathbb{P}^{*}$, we will use a superscript $*$ when working under $\mathbb{P}^{*}$. For example, $\mathbb{E}$ and $\mathbb{E}^{*}$ stand for the expectations under $\mathbb{P}$ and $\mathbb{P}^{*}$, respectively. For what concerns the filtrations, when necessary, we will use the following illustrating notation: $x, X, X^{\tau}$ to denote processes adapted to $\mathbb{F}, \mathbb{G}$ and $\mathbb{G}^{\tau}$, respectively (we shall not use the same notation for processes stopped at $\tau$, so that there will be no possible confusion for the notation $X^{\tau}$ ). 
The following proposition provides a relation between martingales w.r.t. a "larger" and a "smaller" filtration and turns out to be useful in the subsequent sections:

Proposition 2.5. Let $\widetilde{\mathbb{F}}$ be a filtration larger than $\mathbb{F}$, that is $\mathcal{F}_{t} \subseteq \widetilde{\mathcal{F}}_{t}$, for every $t \geq 0$. If $x$ is a u.i. (uniformly integrable) $\mathbb{F}$-martingale, then there exists an $\widetilde{\mathbb{F}}$-martingale $\widetilde{x}$, such that $\mathbb{E}\left(\widetilde{x}_{t} \mid \mathcal{F}_{t}\right)=x_{t}, t \geq 0$.

Proof. The process $\widetilde{x}$ defined by $\widetilde{x}_{t}:=\mathbb{E}\left(x_{\infty} \mid \widetilde{\mathcal{F}}_{t}\right)$ is an $\widetilde{\mathbb{F}}$-martingale, and

$$
\mathbb{E}\left(\widetilde{x}_{t} \mid \mathcal{F}_{t}\right)=\mathbb{E}\left(\mathbb{E}\left(x_{\infty} \mid \widetilde{\mathcal{F}}_{t}\right) \mid \mathcal{F}_{t}\right)=\mathbb{E}\left(x_{\infty} \mid \mathcal{F}_{t}\right)=x_{t}
$$

Remark 2.6. The uniqueness of such a martingale $\widetilde{x}$ is not claimed in the above proposition and does not hold in general.

We now recall the definition of the immersion property, a terminology which will be used in the sequel. Under a given probability measure $\mathbb{Q}$, a filtration $\mathbb{F}$ is said to be immersed in a larger filtration $\widetilde{\mathbb{F}}$, if every $(\mathbb{Q}, \mathbb{F})$-martingale is a $(\mathbb{Q}, \widetilde{\mathbb{F}})$-martingale.

\subsection{Characterization of different measurability properties}

Before focusing on the three topics announced from the beginning, we recall some important results on the characterization of $\mathcal{G}_{t}^{\tau}$ and $\mathcal{G}_{t}$-measurable random variables, as well as $\mathbb{G}^{\tau}$ and $\mathbb{G}$-predictable processes. The necessary part of the result below, in the case of predictable processes, is due to Jeulin [19], Lemma 3.13. See also Yor [25].

Proposition 2.7. One has:

(i) a random variable $Y_{t}^{\tau}$ is $\mathcal{G}_{t}^{\tau}$-measurable if and only if it is of the form $Y_{t}^{\tau}(\omega)=y_{t}(\omega, \tau(\omega))$, for some $\mathcal{F}_{t} \otimes \mathcal{B}\left(\mathbb{R}^{+}\right)$-measurable random variable $y_{t}(\cdot, u)$

(ii) a process $Y^{\tau}$ is $\mathbb{G}^{\tau}$-predictable if and only if it is of the form $Y_{t}^{\tau}(\omega)=y_{t}(\omega, \tau(\omega)), t \geq 0$, where $(t, \omega, u) \mapsto$ $y_{t}(\omega, u)$ is a $\mathcal{P}(\mathbb{F}) \otimes \mathcal{B}\left(\mathbb{R}^{+}\right)$-measurable function.

Proof. The proof of part (i) is based on the fact that $\mathcal{G}_{t}^{\tau}$-measurable random variables are generated by random variables of the form $X_{t}(\omega)=x_{t}(\omega) f(\tau(\omega))$, with $x_{t} \in \mathcal{F}_{t}$ and $f$ bounded Borel function on $\mathbb{R}^{+}$;

(ii) it suffices to notice that processes of the form $X_{t}:=x_{t} f(\tau), t \geq 0$, where $x$ is $\mathbb{F}$-predictable and $f$ is a bounded Borel function on $\mathbb{R}^{+}$, generate the $\mathbb{G}^{\tau}$-predictable $\sigma$-field.

For what concerns the progressive enlargement setting, the following result is analogous to Proposition 2.7. The necessity of part (ii) is already proved in Jeulin [19], Lemma 4.4.

Proposition 2.8. One has:

(i) a random variable $Y_{t}$ is $\mathcal{G}_{t}$-measurable if and only if it is of the form $Y_{t}(\omega)=\widetilde{y}_{t}(\omega) \mathbb{1}_{t<\tau(\omega)}+$ $\widehat{y}_{t}(\omega, \tau(\omega)) \mathbb{1}_{\tau(\omega) \leq t}$ for some $\mathcal{F}_{t}$-measurable random variable $\widetilde{y}_{t}$ and some family of $\mathcal{F}_{t} \otimes \mathcal{B}\left(\mathbb{R}^{+}\right)$-measurable random variables $\widehat{y}_{t}(\cdot, u), t \geq u$;

(ii) a process $Y$ is $\mathbb{G}$-predictable if and only if it is of the form $Y_{t}(\omega)=\widetilde{y}_{t}(\omega) \mathbb{1}_{t \leq \tau(\omega)}+\widehat{y}_{t}(\omega, \tau(\omega)) \mathbb{1}_{\tau(\omega)<t}, t \geq 0$, where $\widetilde{y}$ is $\mathbb{F}$-predictable and $(t, \omega, u) \mapsto \widehat{y}_{t}(\omega, u)$ is a $\mathcal{P}(\mathbb{F}) \otimes \mathcal{B}\left(\mathbb{R}^{+}\right)$-measurable function.

Proof. For part (i), it suffices to recall that $\mathcal{G}_{t}$-measurable random variables are generated by random variables of the form $X_{t}(\omega)=x_{t}(\omega) f(t \wedge \tau(\omega))$, with $x_{t} \in \mathcal{F}_{t}$ and $f$ a bounded Borel function on $\mathbb{R}^{+}$;

(ii) it suffices to notice that $\mathbb{G}$-predictable processes are generated by processes of the form $X_{t}=x_{t} \mathbb{1}_{t \leq \tau}+$ $\widehat{x}_{t} f(\tau) \mathbb{1}_{\tau<t}, t \geq 0$, where $x, \widehat{x}$ are $\mathbb{F}$-predictable and $f$ is a bounded Borel function, defined on $\mathbb{R}^{+}$.

Such a characterization result does not hold for optional processes, in general. We refer to Barlow [4], remark in pages 318 and 319 , for a counterexample. 


\subsection{Expectation and projection tools}

Lemma 2.9. Let $Y_{t}^{\tau}=y_{t}(\tau)$ be a $\mathcal{G}_{t}^{\tau}$-measurable random variable.

(i) If $y_{t}(\tau)$ is $\mathbb{P}$-integrable and $y_{t}(\tau)=0 \mathbb{P}$-a.s. then, for $\nu$-a.e. $u \geq 0, y_{t}(u)=0 \mathbb{P}$-a.s.;

(ii) for $s \leq t$ one has, $\mathbb{P}$-a.s. (or, equivalently, $\mathbb{P}^{*}$-a.s.):

if $y_{t}(\tau)$ is $\mathbb{P}^{*}$-integrable and if $y_{t}(u)$ is $\mathbb{P}$ (or $\left.\mathbb{P}^{*}\right)$-integrable for any $u \geq 0$,

$$
\mathbb{E}^{*}\left(y_{t}(\tau) \mid \mathcal{G}_{s}^{\tau}\right)=\mathbb{E}^{*}\left(y_{t}(u) \mid \mathcal{F}_{s}\right)_{{ }_{u=\tau}}=\mathbb{E}\left(y_{t}(u) \mid \mathcal{F}_{s}\right)_{\left.\right|_{u=\tau}} ;
$$

if $y_{t}(\tau)$ is $\mathbb{P}$-integrable

$$
\mathbb{E}\left(y_{t}(\tau) \mid \mathcal{G}_{s}^{\tau}\right)=\left.\frac{1}{p_{s}(\tau)} \mathbb{E}\left(y_{t}(u) p_{t}(u) \mid \mathcal{F}_{s}\right)\right|_{u=\tau} .
$$

Proof. (i) We have, by applying Fubini-Tonelli's theorem,

$$
0=\mathbb{E}\left(\left|y_{t}(\tau)\right|\right)=\mathbb{E}\left(\mathbb{E}\left(\left|y_{t}(\tau)\right| \mid \mathcal{F}_{t}\right)\right)=\mathbb{E}\left(\int_{0}^{\infty}\left|y_{t}(u)\right| p_{t}(u) \nu(\mathrm{d} u)\right) .
$$

Then $\int_{0}^{\infty}\left|y_{t}(u)\right| p_{t}(u) \nu(\mathrm{d} u)=0 \mathbb{P}$-a.s. and, given that $p_{t}(u)$ is strictly positive for any $u$, we have that, for $\nu$-almost every $u, y_{t}(\cdot, u)=0 \mathbb{P}$-a.s.;

(ii) the first equality in $(2.1)$ is straightforward for elementary random variables of the form $f(\tau) x_{t}$, given the independence between $\tau$ and $\mathcal{F}_{t}$, for any $t \geq 0$. It is extended to $\mathcal{G}_{t}^{\tau}$-measurable r.v's via the monotone class theorem. The second equality follows from the fact that $\mathbb{P}$ and $\mathbb{P}^{*}$ coincide on $\mathcal{F}_{t}$, for any $t \geq 0$.

Equality (2.2) is an immediate consequence of (2.1), since it suffices, by means of (conditional) Bayes' formula, to pass under the measure $\mathbb{P}^{*}$. Namely, for $s<t$, we have

$$
\mathbb{E}\left(y_{t}(\tau) \mid \mathcal{G}_{s}^{\tau}\right)=\frac{\mathbb{E}^{*}\left(y_{t}(\tau) p_{t}(\tau) \mid \mathcal{G}_{s}^{\tau}\right)}{\mathbb{E}^{*}\left(p_{t}(\tau) \mid \mathcal{G}_{s}^{\tau}\right)}=\left.\frac{1}{p_{s}(\tau)} \mathbb{E}\left(y_{t}(u) p_{t}(u) \mid \mathcal{F}_{s}\right)\right|_{u=\tau},
$$

where in the last equality we have used the previous result $(2.1)$ and the fact that $p(\tau)$ is a $\left(\mathbb{P}^{*}, \mathbb{G}^{\tau}\right)$ martingale. Note that if $y_{t}(\tau)$ is $\mathbb{P}$-integrable, then $\mathbb{E}\left(\int_{0}^{\infty}\left|y_{t}(u)\right| p_{t}(u) \nu(\mathrm{d} u)\right)=\mathbb{E}\left(\left|y_{t}(\tau)\right|\right)<\infty$, which implies that $\mathbb{E}\left(\left|y_{t}(u)\right| p_{t}(u)\right)<\infty$.

When working with the progressively enlarged filtration $\mathbb{G}$, it is convenient to introduce the notation $G$ (resp., $G^{*}(\cdot)$ ) for the Azéma supermartingale associated with $\tau$ under the probability measure $\mathbb{P}\left(\right.$ resp., $\left.\mathbb{P}^{*}\right)$. More precisely,

$$
\begin{aligned}
& G_{t}:=\mathbb{P}\left(\tau>t \mid \mathcal{F}_{t}\right)=\int_{t}^{\infty} p_{t}(u) \nu(\mathrm{d} u), \\
& G^{*}(t):=\mathbb{P}^{*}\left(\tau>t \mid \mathcal{F}_{t}\right)=\mathbb{P}^{*}(\tau>t)=\mathbb{P}(\tau>t)=\int_{t}^{\infty} \nu(\mathrm{d} u) .
\end{aligned}
$$

Note, in particular, that $\left(G_{t}\right)_{t \geq 0}$ is an $\mathbb{F}$ supermartingale, whereas $G^{*}(\cdot)$ is a (deterministic) continuous and decreasing function. Furthermore, it is clear that, under the $(\mathcal{E})$-hypothesis and the hypothesis that the support of $\nu$ is $\mathbb{R}^{+}, G$ and $G^{*}(\cdot)$ do not vanish.

Lemma 2.10. Let $Y_{t}^{\tau}=y_{t}(\tau)$ be a $\mathcal{G}_{t}^{\tau}$-measurable, $\mathbb{P}$-integrable random variable. Then, for $s \leq t$,

$$
\mathbb{E}\left(Y_{t}^{\tau} \mid \mathcal{G}_{s}\right)=\mathbb{E}\left(y_{t}(\tau) \mid \mathcal{G}_{s}\right)=\widetilde{y}_{s} \mathbb{1}_{s<\tau}+\widehat{y}_{s}(\tau) \mathbb{1}_{\tau \leq s},
$$

with

$$
\begin{aligned}
\widetilde{y}_{s} & =\frac{1}{G_{s}} \mathbb{E}\left(\int_{s}^{+\infty} y_{t}(u) p_{t}(u) \nu(\mathrm{d} u) \mid \mathcal{F}_{s}\right), \\
\widehat{y}_{s}(u) & =\frac{1}{p_{s}(u)} \mathbb{E}\left(y_{t}(u) p_{t}(u) \mid \mathcal{F}_{s}\right) .
\end{aligned}
$$


Proof. From the above Proposition 2.8, it is clear that $\mathbb{E}\left(y_{t}(\tau) \mid \mathcal{G}_{s}\right)$ can be written in the form $\widetilde{y}_{s} \mathbb{1}_{s<\tau}+$ $\widehat{y}_{s}(\tau) \mathbb{1}_{\tau \leq s}$. On the set $\{s<\tau\}$, we have, applying Lemma 3.1.2 in Bielecki et al. [5] and using the ( $\left.\mathcal{E}\right)$-hypothesis (see also [9] for analogous computations),

$$
\begin{aligned}
\mathbb{1}_{s<\tau} \mathbb{E}\left(y_{t}(\tau) \mid \mathcal{G}_{s}\right) & =\mathbb{1}_{s<\tau} \frac{\mathbb{E}\left[\mathbb{E}\left(y_{t}(\tau) \mathbb{1}_{s<\tau} \mid \mathcal{F}_{t}\right) \mid \mathcal{F}_{s}\right]}{G_{s}} \\
& =\mathbb{1}_{s<\tau} \frac{1}{G_{s}} \mathbb{E}\left(\int_{s}^{+\infty} y_{t}(u) p_{t}(u) \nu(\mathrm{d} u) \mid \mathcal{F}_{s}\right)=: \mathbb{1}_{s<\tau} \widetilde{y}_{s}
\end{aligned}
$$

On the complementary set, we have, by applying Lemma 2.9 ,

$$
\mathbb{1}_{\tau \leq s} \mathbb{E}\left(y_{t}(\tau) \mid \mathcal{G}_{s}\right)=\mathbb{1}_{\tau \leq s} \mathbb{E}\left[\mathbb{E}\left(y_{t}(\tau) \mid \mathcal{G}_{s}^{\tau}\right) \mid \mathcal{G}_{s}\right]=\left.\mathbb{1}_{\tau \leq s} \frac{1}{p_{s}(\tau)} \mathbb{E}\left(y_{t}(u) p_{t}(u) \mid \mathcal{F}_{s}\right)\right|_{u=\tau}=: \mathbb{1}_{\tau \leq s} \widehat{y}_{s}(\tau) .
$$

For $s>t$, we obtain $\mathbb{E}\left(Y_{t}^{\tau} \mid \mathcal{G}_{s}\right)=\frac{1}{G_{s}} \int_{s}^{\infty} y_{t}(u) p_{s}(u) \nu(\mathrm{d} u) \mathbb{1}_{s<\tau}+y_{t}(\tau) \mathbb{1}_{\tau \leq s}$.

As an application, projecting the martingale $L$ (defined earlier as $L_{t}=\frac{1}{p_{t}(\tau)}, t \geq 0$ ) on $\mathbb{G}$ yields to the corresponding Radon-Nikodým density on $\mathbb{G}$ :

$$
\mathrm{dP}^{*}{ }_{\mid \mathcal{G}_{t}}=\ell_{t} \mathrm{~d} \mathbb{P}_{\mid \mathcal{G}_{t}},
$$

with

$$
\begin{aligned}
\ell_{t} & :=\mathbb{E}\left(L_{t} \mid \mathcal{G}_{t}\right)=\mathbb{1}_{t<\tau} \frac{1}{G_{t}} \int_{t}^{\infty} \nu(\mathrm{d} u)+\mathbb{1}_{\tau \leq t} \frac{1}{p_{t}(\tau)} \\
& =\mathbb{1}_{t<\tau} \frac{G^{*}(t)}{G_{t}}+\mathbb{1}_{\tau \leq t} \frac{1}{p_{t}(\tau)} .
\end{aligned}
$$

We now recall some useful facts concerning the compensated martingale of the default indicator process $H_{t}=$ $\mathbb{1}_{\tau \leq t}, t \geq 0$. We know, from the general theory (see, for example, [9]), that the process $M$ defined as

$$
M_{t}:=H_{t}-\int_{0}^{t \wedge \tau} \lambda_{s} \nu(\mathrm{d} s), \quad t \geq 0
$$

with $\lambda_{t}=\frac{p_{t}(t)}{G_{t}}$, is a $(\mathbb{P}, \mathbb{G})$-martingale and that

$$
M_{t}^{*}:=H_{t}-\int_{0}^{t \wedge \tau} \lambda^{*}(s) \nu(\mathrm{d} s), \quad t \geq 0
$$

with $\lambda^{*}(t)=\frac{1}{G^{*}(t)}$, is a $\left(\mathbb{P}^{*}, \mathbb{G}\right)$-martingale. Furthermore, since $\lambda^{*}$ is deterministic, $M^{*}$ (being $\mathbb{H}$-adapted) is a $\left(\mathbb{P}^{*}, \mathbb{H}\right)$-martingale, too.

We conclude this subsection with the following two propositions, concerning the predictable projection, respectively on $\mathbb{F}$ and on $\mathbb{G}$, of a $\mathbb{G}^{\tau}$-predictable process. The first result is due to Jacod [15], Lemma 1.10.

Proposition 2.11. Let $Y^{\tau}=y(\tau)$ be a $\mathbb{G}^{\tau}$-predictable, positive or bounded, process. Then, the $\mathbb{P}$-predictable projection of $Y^{\tau}$ on $\mathbb{F}$ is given by

$$
{ }^{(p)}\left(Y^{\tau}\right)_{t}=\int_{0}^{\infty} y_{t}(u) p_{t-}(u) \nu(\mathrm{d} u) .
$$

Proof. It is obtained by a monotone class argument and by using the definition of density of $\tau$, writing, for "elementary" processes, $Y_{t}^{\tau}:=y_{t} f(\tau)$, with $y$ a bounded $\mathbb{F}$-predictable process and $f$ a bounded Borel function. For this, we refer to the proof of Lemma 1.10 in [15]. 
Proposition 2.12. Let $Y^{\tau}=y(\tau)$ be a $\mathbb{G}^{\tau}$-predictable, positive or bounded, process. Then, the $\mathbb{P}$-predictable projection of $Y^{\tau}$ on $\mathbb{G}$ is given by

$$
{ }^{(p)}\left(Y^{\tau}\right)_{t}=\mathbb{1}_{t \leq \tau} \frac{1}{G_{t-}} \int_{t}^{\infty} y_{t}(u) p_{t-}(u) \nu(\mathrm{d} u)+\mathbb{1}_{\tau<t} y_{t}(\tau) .
$$

Proof. In this proof, for clarity, the left-hand side superscript " $(p \mathbb{G})$ " denotes the $\mathbb{P}$-predictable projection on $\mathbb{G}$, while the left-hand side superscript " $(p \mathbb{F})$ " indicates the $\mathbb{P}$-predictable projection on $\mathbb{F}$. By the definition of predictable projection, we know (from Prop. 2.8 (ii)) that we are looking for a (unique) process of the form

$$
{ }^{(p \mathbb{G})}\left(Y^{\tau}\right)_{t}=\widetilde{y}_{t} \mathbb{1}_{t \leq \tau}+\widehat{y}_{t}(\tau) \mathbb{1}_{\tau<t}, \quad t \geq 0,
$$

where $\widetilde{y}$ is $\mathbb{F}$-predictable, positive or bounded, and $(t, \omega, u) \mapsto \widehat{y}_{t}(\omega, u)$ is a $\mathcal{P}(\mathbb{F}) \otimes \mathcal{B}\left(\mathbb{R}^{+}\right)$-measurable positive or bounded function, to be identified.

- On the predictable set $\{\tau<t\}$, being $Y^{\tau}$ a $\mathbb{G}^{\tau}$-predictable, positive or bounded, process (recall Prop. 2.7 (ii)), we immediately find $\widehat{y}(\tau)=y(\tau)$;

- on the complementary set $\{t \leq \tau\}$, introducing the $\mathbb{G}$-predictable process

$$
Y:={ }^{(p \mathbb{G})}\left(Y^{\tau}\right)
$$

it is possible to use Remark 4.5, page 64 of Jeulin [19] (see also Dellacherie and Meyer [8], Chap. XX, p. 186), to write

$$
Y \mathbb{1}_{\rrbracket 0, \tau \rrbracket}=\frac{1}{G_{-}}{ }^{(p \mathbb{F})}\left(Y \mathbb{1}_{\rrbracket 0, \tau \rrbracket}\right) \mathbb{1}_{\rrbracket 0, \tau \rrbracket}=\frac{1}{G_{-}} \quad(p \mathbb{F})\left({ }^{(p \mathbb{G})}\left(Y^{\tau}\right) \mathbb{1}_{\rrbracket 0, \tau \rrbracket}\right) \mathbb{1}_{\rrbracket 0, \tau \rrbracket} .
$$

We then have, being $\mathbb{1}_{\rrbracket 0, \tau \rrbracket}$, by definition, $\mathbb{G}$-predictable (recall that $\tau$ is a $\mathbb{G}$-stopping time),

$$
Y \mathbb{1}_{\rrbracket 0, \tau \rrbracket}=\frac{1}{G_{-}} \quad{ }^{(p \mathbb{F})}\left(Y^{\tau} \mathbb{1}_{\rrbracket 0, \tau \rrbracket}\right) \mathbb{1}_{\rrbracket 0, \tau \rrbracket},
$$

where the last equality follows by the definition of predictable projection, being $\mathbb{F} \subset \mathbb{G}$. Finally, given the result in Proposition 2.11, we have

$$
{ }^{(p \mathbb{F})}\left(Y^{\tau} \mathbb{1}_{\rrbracket 0, \tau \rrbracket}\right)_{t}=\int_{t}^{+\infty} y_{t}(u) p_{t-}(u) \nu(\mathrm{d} u)
$$

and the proposition is proved.

\section{Martingales' CharaCterization}

The aim of this section is to characterize $\left(\mathbb{P}, \mathbb{G}^{\tau}\right)$ and $(\mathbb{P}, \mathbb{G})$-martingales in terms of $(\mathbb{P}, \mathbb{F})$-martingales.

Proposition 3.1 (characterization of $\left(\mathbb{P}, \mathbb{G}^{\tau}\right)$-martingales in terms of $(\mathbb{P}, \mathbb{F})$-martingales). A process $Y^{\tau}=$ $y(\tau)$ is a $\left(\mathbb{P}, \mathbb{G}^{\tau}\right)$-martingale if and only if $\left(y_{t}(u) p_{t}(u), t \geq 0\right)$ is a $(\mathbb{P}, \mathbb{F})$-martingale, for $\nu$-almost every $u \geq 0$.

Proof. The sufficiency is a direct consequence of Proposition 2.7 and Lemma 2.9 (ii).

Conversely, assume that $y(\tau)$ is a $\mathbb{G}^{\tau}$-martingale. Then, for $s \leq t$, from Lemma 2.9 (ii),

$$
y_{s}(\tau)=\mathbb{E}\left(y_{t}(\tau) \mid \mathcal{G}_{s}^{\tau}\right)=\frac{1}{p_{s}(\tau)} \mathbb{E}\left(y_{t}(u) p_{t}(u) \mid \mathcal{F}_{s}\right)_{\mid u=\tau}
$$

and the result follows from Lemma 2.9 (i). 
Remark 3.2. This result, being a consequence of the Girsanov theorem ( $c f$. the proof of Lem. 2.9 (ii)), can immediately be extended to $\left(\mathbb{P}, \mathbb{G}^{\tau}\right)$-local martingales.

Passing to the progressive enlargement setting, we state and prove a martingale characterization result, first formulated by El Karoui et al. in [9], Theorem 5.7.

Proposition 3.3 (characterization of $(\mathbb{P}, \mathbb{G}$ )-martingales in terms of $(\mathbb{P}, \mathbb{F})$-martingales). $A \mathbb{G}$-adapted process $Y_{t}:=\widetilde{y}_{t} \mathbb{1}_{t<\tau}+\widehat{y}_{t}(\tau) \mathbb{1}_{\tau \leq t}, t \geq 0$, is a $(\mathbb{P}, \mathbb{G})$-martingale if and only if the following two conditions are satisfied

(i) for $\nu$-almost every $u \geq 0,\left(\widehat{y}_{t}(u) p_{t}(u), t \geq u\right)$ is a $(\mathbb{P}, \mathbb{F})$-martingale;

(ii) the process $m=\left(m_{t}, t \geq 0\right)$, given by

$$
m_{t}:=\mathbb{E}\left(Y_{t} \mid \mathcal{F}_{t}\right)=\widetilde{y}_{t} G_{t}+\int_{0}^{t} \widehat{y}_{t}(u) p_{t}(u) \nu(\mathrm{d} u),
$$

is a $(\mathbb{P}, \mathbb{F})$-martingale.

Proof. For the necessity, in a first step, we show that we can reduce our attention to the case where $Y$ is u.i.: indeed, let $Y$ be a $(\mathbb{P}, \mathbb{G})$-martingale. For any $T$, let $Y^{(T)}=\left(Y_{t \wedge T}, t \geq 0\right)$ be the associated stopped martingale, which is u.i. Assuming that the result is established for u.i. martingales will prove that the processes in $(i)$ and (ii) are martingales up to time $T$. Since $T$ can be chosen as large as possible, we shall have the result.

Assume, then, that $Y$ is a u.i. $(\mathbb{P}, \mathbb{G})$-martingale. From Proposition 2.5, $Y_{t}=\mathbb{E}\left(Y_{t}^{\tau} \mid \mathcal{G}_{t}\right)$ for some $\left(\mathbb{P}, \mathbb{G}^{\tau}\right)$ martingale $Y^{\tau}$. Proposition 3.1, then, implies that $Y_{t}^{\tau}=y_{t}(\tau)$, where for $\nu$-almost every $u \geq 0$ the process $\left(y_{t}(u) p_{t}(u), t \geq 0\right)$ is a $(\mathbb{P}, \mathbb{F})$-martingale. One then has

$$
\mathbb{1}_{\tau \leq t} \widehat{y}_{t}(\tau)=\mathbb{1}_{\tau \leq t} Y_{t}=\mathbb{1}_{\tau \leq t} \mathbb{E}\left(Y_{t}^{\tau} \mid \mathcal{G}_{t}\right)=\mathbb{E}\left(\mathbb{1}_{\tau \leq t} Y_{t}^{\tau} \mid \mathcal{G}_{t}\right)=\mathbb{1}_{\tau \leq t} y_{t}(\tau),
$$

which implies, in view of Lemma $2.9(\mathrm{i})$, that for $\nu$-almost every $u \leq t$, the identity $y_{t}(u)=\widehat{y}_{t}(u)$ holds $\mathbb{P}$-almost surely. So, $(i)$ is proved. Moreover, $Y$ being a $(\mathbb{P}, \mathbb{G}$ )-martingale, its projection on the smaller filtration $\mathbb{F}$, namely the process $m$ in $(3.1)$, is a $(\mathbb{P}, \mathbb{F})$-martingale.

Conversely, assuming $(i)$ and $(i i)$, we verify that $\mathbb{E}\left(Y_{t} \mid \mathcal{G}_{s}\right)=Y_{s}$ for $s \leq t$. We start by noting that

$$
\mathbb{E}\left(Y_{t} \mid \mathcal{G}_{s}\right)=\mathbb{1}_{s<\tau} \frac{1}{G_{s}} \mathbb{E}\left(Y_{t} \mathbb{1}_{s<\tau} \mid \mathcal{F}_{s}\right)+\mathbb{1}_{\tau \leq s} \mathbb{E}\left(Y_{t} \mathbb{1}_{\tau \leq s} \mid \mathcal{G}_{s}\right) .
$$

We then compute the two conditional expectations in (3.2):

$$
\begin{aligned}
\mathbb{E}\left(Y_{t} \mathbb{1}_{s<\tau} \mid \mathcal{F}_{s}\right) & =\mathbb{E}\left(Y_{t} \mid \mathcal{F}_{s}\right)-\mathbb{E}\left(Y_{t} \mathbb{1}_{\tau \leq s} \mid \mathcal{F}_{s}\right) \\
& =\mathbb{E}\left(m_{t} \mid \mathcal{F}_{s}\right)-\mathbb{E}\left(\mathbb{E}\left(\widehat{y}_{t}(\tau) \mathbb{1}_{\tau \leq s} \mid \mathcal{F}_{t}\right) \mid \mathcal{F}_{s}\right) \\
& =m_{s}-\mathbb{E}\left(\int_{0}^{s} \widehat{y}_{t}(u) p_{t}(u) \nu(\mathrm{d} u) \mid \mathcal{F}_{s}\right) \\
& =\widetilde{y}_{s} G_{s}+\int_{0}^{s} \widehat{y}_{s}(u) p_{s}(u) \nu(\mathrm{d} u)-\int_{0}^{s} \widehat{y}_{s}(u) p_{s}(u) \nu(\mathrm{d} u)=\widetilde{y}_{s} G_{s}
\end{aligned}
$$

where we used Fubini-Tonelli's theorem and the condition $(i)$ to obtain the next-to-last identity.

Also, an application of Lemma 2.10 yields to

$$
\begin{aligned}
\mathbb{E}\left(Y_{t} \mathbb{1}_{\tau \leq s} \mid \mathcal{G}_{s}\right) & =\mathbb{E}\left(\widehat{y}_{t}(\tau) \mathbb{1}_{\tau \leq s} \mid \mathcal{G}_{s}\right)=\left.\mathbb{1}_{\tau \leq s} \frac{1}{p_{s}(\tau)} \mathbb{E}\left(\widehat{y}_{t}(u) p_{t}(u) \mid \mathcal{F}_{s}\right)\right|_{u=\tau} \\
& =\mathbb{1}_{\tau \leq s} \frac{1}{p_{s}(\tau)} \widehat{y}_{s}(\tau) p_{s}(\tau)=\mathbb{1}_{\tau \leq s} \widehat{y}_{s}(\tau)
\end{aligned}
$$

where the next-to-last identity holds in view of the condition (ii). 
Remark 3.4. The extension of this characterization result to local martingales is more difficult. Nevertheless, the sufficient condition holds: if $\left(\widehat{y}_{t}(u) p_{t}(u), t \geq u\right)$ and $\left(\widetilde{y}_{t} G_{t}+\int_{0}^{t} \widehat{y}_{t}(u) p_{t}(u) \nu(\mathrm{d} u), t \geq 0\right)$ are $(\mathbb{P}, \mathbb{F})$-local martingales, then using $\mathbb{F}$-stopping times $T_{n}$ for localization, one gets that $\left(Y_{t \wedge T_{n}}, t \geq 0\right)$ is a $(\mathbb{P}, \mathbb{G})$-martingale, hence $Y$ is a $(\mathbb{P}, \mathbb{G})$-local martingale.

\section{CAnonical DeCOMposition}

In this section, we work under $\mathbb{P}$ and we show that any $\mathbb{F}$-local martingale $x$ is a semimartingale in the initially enlarged filtration $\mathbb{G}^{\tau}$ and in the progressively enlarged filtration $\mathbb{G}$, and that any $\mathbb{G}$-local martingale is a $\mathbb{G}^{\tau}$ semimartingale. We also provide the canonical decomposition of any $\mathbb{F}$-local martingale as a semimartingale in $\mathbb{G}^{\tau}$ and in $\mathbb{G}$. Under the assumption that the $\mathbb{F}$-conditional law of $\tau$ is absolutely continuous w.r.t. the law of $\tau$, these questions were answered by Jacod in [15], in the initial enlargement setting, and in Jeanblanc and Le Cam [16], in the progressive enlargement case. Our aim here is to recover their results in an alternative manner, under the $(\mathcal{E})$-hypothesis.

We will need the following technical result, concerning the existence of the predictable bracket $\langle x, p .(u)\rangle$. From Theorem 2.5.a in [15], it follows immediately that, under the $(\mathcal{E})$-hypothesis, for every $(\mathbb{P}, \mathbb{F})$-(local) martingale $x$, there exists a $\nu$-negligible set $B$ (depending on $x$ ), such that $\langle x, p$. $(u)\rangle$ is well-defined for $u \notin B$. Hereafter, by $\langle x, p .(\tau)\rangle$ we mean $\left.\langle x, p .(u)\rangle\right|_{u=\tau}$.

Furthermore, according to Theorem 2.5.b in [15], under the $(\mathcal{E})$-hypothesis, there exists an $\mathbb{F}$-predictable increasing process $A$ and a $\mathcal{P}(\mathbb{F}) \otimes \mathcal{B}\left(\mathbb{R}^{+}\right)$-measurable function $(t, \omega, u) \rightarrow k_{t}(\omega, u)$ such that, for any $u \notin B$ and for all $t \geq 0$,

$$
\langle x, p .(u)\rangle_{t}=\int_{0}^{t} k_{s}(u) p_{s-}(u) \mathrm{d} A_{s} \quad \text { a.s. }
$$

(the two processes $A$ and $k$ depend on $x$, however, to simplify the notation, we do not write $A^{(x)}$, nor $k^{(x)}$ ).

Moreover,

$$
\int_{0}^{t}\left|k_{s}(\tau)\right| \mathrm{d} A_{s}<\infty \quad \text { a.s., for any } t>0 \text {. }
$$

The following two propositions provide, under the $(\mathcal{E})$-hypothesis, the canonical decomposition of any $(\mathbb{P}, \mathbb{F})$ local martingale $x$ in the enlarged filtrations $\mathbb{G}^{\tau}$ and $\mathbb{G}$, respectively. The case of initial enlargement has been essentially established by Jacod (see Thm. 2.5.c in [15]), using a direct verification. In our setting, one can obtain Jacod's result using the equivalent change of probability measure methodology (see also Amendinger [1]). Indeed, in view of Remark 2.3, if $x$ is a $\left(\mathbb{P}, \mathbb{F}\right.$ )-local martingale, it is a $\left(\mathbb{P}^{*}, \mathbb{G}^{\tau}\right)$-local martingale, too. Noting that $\frac{\mathrm{d} \mathbb{P}}{\mathrm{dP}}=p_{t}(\tau)$ on $\mathcal{G}_{t}^{\tau}$, Girsanov's theorem tells us that the process $X^{\tau}$, defined by

$$
X_{t}^{\tau}:=x_{t}-\int_{0}^{t} \frac{\mathrm{d}\langle x, p .(\tau)\rangle_{s}}{p_{s-}(\tau)}
$$

is a $\left(\mathbb{P}, \mathbb{G}^{\tau}\right)$-local martingale. However, the proof presented here (for Prop. 4.1), is based on the $\left(\mathbb{P}, \mathbb{G}^{\tau}\right)$ martingales' characterization result given in Proposition 3.1.

Proposition 4.1 (canonical decomposition in $\left.\mathbb{G}^{\tau}\right)$. Any $(\mathbb{P}, \mathbb{F})$-local martingale $x$ is a $\left(\mathbb{P}, \mathbb{G}^{\tau}\right)$-semimartingale with canonical decomposition

$$
x_{t}=X_{t}^{\tau}+\int_{0}^{t} \frac{\mathrm{d}\langle x, p .(\tau)\rangle_{s}}{p_{s-}(\tau)}
$$

where $X^{\tau}$ is a $\left(\mathbb{P}, \mathbb{G}^{\tau}\right)$-local martingale. 
Proof. In view of Proposition 3.1 and Remark 3.2, using the notation $X^{\tau}=x(\tau)$, it suffices to show that, for $\nu$-almost every $u \geq 0$, the process

$$
x_{t}(u) p_{t}(u):=\left(x_{t}-\int_{0}^{t} \frac{\mathrm{d}\langle x, p .(u)\rangle_{s}}{p_{s-}(u)}\right) p_{t}(u), \quad t \geq 0,
$$

is a $(\mathbb{P}, \mathbb{F})$-local martingale. Indeed, integration by parts formula leads to

$$
\mathrm{d}\left(x_{t}(u) p_{t}(u)\right)=p_{t-}(u) \mathrm{d} x_{t}+x_{t-}(u) \mathrm{d} p_{t}(u)+\mathrm{d}\left([x(u), p(u)]_{t}-\langle x(u), p(u)\rangle_{t}\right) .
$$

Hence, being the sum of three $(\mathbb{P}, \mathbb{F})$-local martingales, the process $x(u) p(u)$ is a $(\mathbb{P}, \mathbb{F})$-local martingale.

Now, any $(\mathbb{P}, \mathbb{F})$-local martingale is a $\mathbb{G}$-adapted process and a $\left(\mathbb{P}, \mathbb{G}^{\tau}\right)$-semimartingale (from the above Prop. 4.1), so in view of Stricker's theorem in [23], it is also a $\mathbb{G}$-semimartingale. The following proposition aims to obtain the $\mathbb{G}$-canonical decomposition of an $\mathbb{F}$-local martingale. We refer to [16] for an alternative proof.

In order to study the canonical decomposition in $\mathbb{G}$, we add a regularity condition.

Assumption 4.2. There exists a version of the process $\left(p_{t}(t), t \geq 0\right)$, such that $(\omega, t) \rightarrow p_{t}(\omega, t)$ is $\mathcal{F}_{t} \otimes \mathcal{B}\left(\mathbb{R}^{+}\right)$measurable.

Then, the Azéma supermartingale $G$, introduced in equation (2.3), admits the Doob-Meyer decomposition $G_{t}=\mu_{t}-\int_{0}^{t} p_{u}(u) \nu(\mathrm{d} u), t \geq 0$, where $\mu$ is the $\mathbb{F}$-martingale defined as

$$
\mu_{t}:=1-\int_{0}^{t}\left(p_{t}(u)-p_{u}(u)\right) \nu(\mathrm{d} u)
$$

(see, e.g., Sect. 4.2 .1 in [9]).

Before passing to the rigorous result on the canonical decomposition in $\mathbb{G}$, one can guess the form of the decomposition by means of a heuristic argument, based on the equivalent change of probability measure: $(\mathbb{P}, \mathbb{F})$ local martingale $x$ being a $\left(\mathbb{P}^{*}, \mathbb{G}\right)$-local martingale,

$$
x_{t}-\int_{0}^{t} \frac{1}{\ell_{s-}^{*}} \mathrm{~d}\left\langle x, \ell^{*}\right\rangle_{s}
$$

is a $(\mathbb{P}, \mathbb{G})$-local martingale, where $\ell^{*}:=\frac{1}{\ell}$ is the Radon-Nikodým density of $\mathbb{P}$ w.r.t. $\mathbb{P}^{*}$, given by

$$
\left.\frac{\mathrm{d} \mathbb{P}}{\mathrm{d} \mathbb{P}^{*}}\right|_{\mathcal{G}_{t}}=\mathbb{1}_{t<\tau} \frac{G_{t}}{G^{*}(t)}+\mathbb{1}_{\tau \leq t} p_{t}(\tau)=\frac{1}{\ell_{t}}=\ell_{t}^{*}
$$

Based on the form of $\ell^{*}$, one has

$$
\mathbb{1}_{s<\tau} \mathrm{d}\left\langle x, \ell^{*}\right\rangle_{s}=\mathbb{1}_{s<\tau} \frac{\mathrm{d}\langle x, G\rangle_{s}}{G^{*}(s)} .
$$

This observation suggests Proposition 4.3 below, the proof of which is based on the $(\mathbb{P}, \mathbb{G}$ )-martingales' characterization result presented in Section 3.

Proposition 4.3 (canonical decomposition in $\mathbb{G})$. Any (càdlàg) $(\mathbb{P}, \mathbb{F})$-local martingale $x$ is a $(\mathbb{P}, \mathbb{G})$ semimartingale with canonical decomposition

$$
x_{t}=X_{t}+\int_{0}^{t \wedge \tau} \frac{\mathrm{d}\langle x, G\rangle_{s}}{G_{s-}}+\int_{t \wedge \tau}^{t} \frac{\mathrm{d}\langle x, p .(\tau)\rangle_{s}}{p_{s-}(\tau)},
$$

where $X$ is a $(\mathbb{P}, \mathbb{G})$-local martingale. 
Proof. Relying on Remark 3.4, we check that $X$, defined in (4.3), is a $(\mathbb{P}, \mathbb{G})$-local martingale. We note that $X_{t}=\mathbb{1}_{t<\tau} \widetilde{x}_{t}+\mathbb{1}_{\tau \leq t} \widehat{x}_{t}(\tau)$ with

$$
\widetilde{x}_{t}=x_{t}-\int_{0}^{t} \frac{\mathrm{d}\langle x, G\rangle_{s}}{G_{s-}}, \quad \widehat{x}_{t}(u)=x_{t}-\int_{0}^{u} \frac{\mathrm{d}\langle x, G\rangle_{s}}{G_{s-}}-\int_{u}^{t} \frac{\mathrm{d}\langle x, p .(u)\rangle_{s}}{p_{s-}(u)} .
$$

We have to verify that

(i) $\left(\widehat{x}_{t}(u) p_{t}(u), t \geq u\right)$ is a $(\mathbb{P}, \mathbb{F})$-local martingale;

(ii) $\left(\widetilde{x}_{t} G_{t}+\int_{0}^{t} \widehat{x}_{t}(u) p_{t}(u) \nu(\mathrm{d} u), t \geq 0\right)$ is a $(\mathbb{P}, \mathbb{F})$-local martingale.

In the proof of Proposition 4.1, we verified that (i) holds. In order to show (ii), we apply Itô's formula

$$
\mathrm{d}\left(\widetilde{x}_{t} G_{t}+\int_{0}^{t} \widehat{x}_{t}(u) p_{t}(u) \nu(\mathrm{d} u)\right)=G_{t-} \mathrm{d} x_{t}+d\left([x, G]_{t}-\langle x, G\rangle_{t}\right)+\widetilde{x}_{t-} \mathrm{d} \mu_{t}+\left(\widehat{x}_{t}(t)-\widetilde{x}_{t-}\right) p_{t}(t) \nu(\mathrm{d} t)+\mathrm{d} z_{t},
$$

where $z_{t}:=\int_{s=0}^{t} \int_{u=0}^{s} \nu(\mathrm{d} u) \mathrm{d} \zeta_{s}(u)$ and $\zeta_{t}(u):=\widehat{x}_{t}(u) p_{t}(u)$. The first three terms on the righthand-side of (4.4) are $(\mathbb{P}, \mathbb{F})$-local martingales, the fourth term is zero since $\widehat{x}_{t}(t)-\widetilde{x}_{t-}=\Delta \widetilde{x}_{t}$, and the $\mathbb{F}$-adapted process $\widetilde{x}$ has no jump at time $\tau$ (because, in our setting, $\tau$ avoids $\mathbb{F}$-stopping times). So, if we show that $z$ is a $(\mathbb{P}, \mathbb{F}$ )-local martingale, we are done. To do this, applying Fubini-Tonelli's theorem, one has

$$
z_{t}=\int_{u=0}^{t} \int_{s=u}^{t} \mathrm{~d} \zeta_{s}(u) \nu(\mathrm{d} u)=\int_{0}^{t}\left(\zeta_{t}(u)-\zeta_{u}(u)\right) \nu(\mathrm{d} u) .
$$

So, for $t_{0}<t$, one has

$$
z_{t}=\int_{0}^{t_{0}}\left(\zeta_{t}(u)-\zeta_{u}(u)\right) \nu(\mathrm{d} u)+\int_{t_{0}}^{t}\left(\zeta_{t}(u)-\zeta_{u}(u)\right) \nu(\mathrm{d} u)
$$

Now, if $\left(\zeta_{t}(u), t \geq 0\right)$ is a $(\mathbb{P}, \mathbb{F})$-martingale, one gets $\mathbb{E}\left(z_{t} \mid \mathcal{F}_{t_{0}}\right)=z_{t_{0}}$. In the case where $\zeta$. $(u)$ is a $(\mathbb{P}, \mathbb{F})$-local martingale the result is achieved by means of a localization argument.

Remark 4.4. In a recent paper, Kchia et al. [20] have obtained the same decomposition formula, using projection tools, in a more general setting. The main challenge in their approach is that if a $\mathbb{G}^{\tau}$-local martingale is $\mathbb{G}$-adapted, it is not necessarily a $\mathbb{G}$-local martingale (as remarked also by Stricker [23]).

The following lemma provides a formula for the predictable quadratic covariation process $\langle x, G\rangle=\langle x, \mu\rangle$ in terms of the density $p$.

Proposition 4.5. Let $x$ be a $(\mathbb{P}, \mathbb{F})$-local martingale and $\mu$ the $\mathbb{F}$-martingale part in the Doob-Meyer decomposition of $G$. If $k p_{-}$is $\mathrm{d} A \otimes \mathrm{d} \nu$-integrable, then

$$
\langle x, \mu\rangle_{t}=\int_{0}^{t} \mathrm{~d} A_{s} \int_{s}^{\infty} \nu(\mathrm{d} u) k_{s}(u) p_{s-}(u)
$$

where $k$ was introduced in equation (4.1).

Proof. First consider the right-hand side of (4.5), that is, by definition, predictable, and apply Fubini-Tonelli's theorem

$$
\begin{aligned}
\xi_{t} & :=\int_{0}^{t} \mathrm{~d} A_{s} \int_{s}^{\infty} k_{s}(u) p_{s-}(u) \nu(\mathrm{d} u) \\
& =\int_{0}^{t} \mathrm{~d} A_{s} \int_{s}^{t} k_{s}(u) p_{s-}(u) \nu(\mathrm{d} u)+\int_{0}^{t} \mathrm{~d} A_{s} \int_{t}^{\infty} k_{s}(u) p_{s-}(u) \nu(\mathrm{d} u) \\
& =\int_{0}^{t} \nu(\mathrm{d} u) \int_{0}^{u} k_{s}(u) p_{s-}(u) \mathrm{d} A_{s}+\int_{t}^{\infty} \nu(\mathrm{d} u) \int_{0}^{t} k_{s}(u) p_{s-}(u) \mathrm{d} A_{s} \\
& =\int_{0}^{t}\langle x, p \cdot(u)\rangle_{u} \nu(\mathrm{d} u)+\int_{t}^{\infty}\langle x, p \cdot(u)\rangle_{t} \nu(\mathrm{d} u) \\
& =\int_{0}^{\infty}\langle x, p \cdot(u)\rangle_{t} \nu(\mathrm{d} u)+\int_{0}^{t}\left(\langle x, p \cdot(u)\rangle_{u}-\langle x, p \cdot(u)\rangle_{t}\right) \nu(\mathrm{d} u) .
\end{aligned}
$$


To verify (4.5), it suffices to show that the process $x \mu-\xi$ is an $\mathbb{F}$-local martingale (since $\xi$ is a predictable, finite variation process). By definition, for $\nu$-almost every $u \in \mathbb{R}^{+}$, the process $\left(m_{t}(u):=x_{t} p_{t}(u)-\langle x, p .(u)\rangle_{t}, t \geq 0\right)$ is an $\mathbb{F}$-local martingale. Then, given that $1=\int_{0}^{\infty} p_{t}(u) \nu(\mathrm{d} u)$ for every $t \geq 0$, a.s., we have

$$
\begin{aligned}
x_{t} \mu_{t}-\xi_{t}= & x_{t} \int_{0}^{\infty} p_{t}(u) \nu(\mathrm{d} u)-x_{t} \int_{0}^{t}\left(p_{t}(u)-p_{u}(u)\right) \nu(\mathrm{d} u) \\
& -\int_{0}^{\infty}\langle x, p \cdot(u)\rangle_{t} \nu(\mathrm{d} u)+\int_{0}^{t}\left(\langle x, p \cdot(u)\rangle_{t}-\langle x, p \cdot(u)\rangle_{u}\right) \nu(\mathrm{d} u) \\
= & \int_{0}^{\infty} m_{t}(u) \nu(\mathrm{d} u)-\int_{0}^{t}\left(m_{t}(u)-m_{u}(u)\right) \nu(\mathrm{d} u)+x_{t} \int_{0}^{t} p_{u}(u) \nu(\mathrm{d} u)-\int_{0}^{t} p_{u}(u) x_{u} \nu(\mathrm{d} u) .
\end{aligned}
$$

The first two terms are local martingales, in view of the martingale property of $m(u)$. As for the last term, using the fact that $\nu$ has no atoms, we find

$$
\begin{aligned}
\mathrm{d}\left(x_{t} \int_{0}^{t} p_{u}(u) \nu(\mathrm{d} u)-\int_{0}^{t} p_{u}(u) x_{u} \nu(\mathrm{d} u)\right) & =\left(\int_{0}^{t} p_{u}(u) \nu(\mathrm{d} u)\right) \mathrm{d} x_{t}+x_{t} p_{t}(t) \nu(\mathrm{d} t)-p_{t}(t) x_{t} \nu(\mathrm{d} t) \\
& =\left(\int_{0}^{t} p_{u}(u) \nu(\mathrm{d} u)\right) \mathrm{d} x_{t}
\end{aligned}
$$

and we have, indeed, proved that $x \mu-\xi$ is an $\mathbb{F}$-local martingale.

We end this section proving that any $\left(\mathbb{P}^{*}, \mathbb{G}\right)$-martingale remains a $\left(\mathbb{P}^{*}, \mathbb{G}^{\tau}\right)$-semimartingale, but it is not necessarily a $\left(\mathbb{P}^{*}, \mathbb{G}^{\tau}\right)$-martingale. Indeed, we have the following result.

Proposition 4.6. Any $\left(\mathbb{P}^{*}, \mathbb{G}\right)$-martingale $Y^{*}$ is a $\left(\mathbb{P}^{*}, \mathbb{G}^{\tau}\right)$ - semimartingale which can have a non-null bounded variation part.

Proof. The result follows immediately from Proposition 3.3 (under $\mathbb{P}^{*}$ ), noticing that the $\left(\mathbb{P}^{*}, \mathbb{G}\right.$ )-martingale $Y^{*}$ can be written as $Y_{t}^{*}=\widetilde{y}_{t}^{*} \mathbb{1}_{t<\tau}+\widehat{y}_{t}^{*}(\tau) \mathbb{1}_{\tau \leq t}$. Therefore, in the filtration $\mathbb{G}^{\tau}$, it is the sum of two $\mathbb{G}^{\tau}-$ semimartingales: the processes $\mathbb{1}_{t<\tau}$ and $\mathbb{1}_{\tau \leq t}$ are $\mathbb{G}^{\tau}$-semimartingales, as well as the processes $\widetilde{y}, \widehat{y}^{*}(\tau)$. Indeed, from Proposition 3.3, recalling that the $\left(\mathbb{P}^{*}, \mathbb{F}\right)$-density of $\tau$ is a constant equal to one, we know that, for every $u>0,\left(\widehat{y}_{t}^{*}(u), t \geq u\right)$ is an $\mathbb{F}$-martingale and that the process $\left(\widetilde{y}_{t}^{*} G^{*}(t)+\int_{0}^{t} \widehat{y}_{u}^{*}(u) \nu(\mathrm{d} u), t \geq 0\right)$ is an $\mathbb{F}$-martingale, hence $\widetilde{y}^{*}$ is a $\mathbb{G}$-semimartingale.

It can be noticed that the $\left(\mathbb{P}^{*}, \mathbb{G}\right)$-martingale $M^{*}$, defined in $(2.6)$, is such that $M_{t}^{*}$ is, for any $t$, a $\mathcal{G}_{0}^{\tau}$ measurable random variable. Therefore, $M^{*}$ is not a $\left(\mathbb{P}^{*}, \mathbb{G}^{\tau}\right)$-martingale, since, for $s \leq t, \mathbb{E}\left(M_{t}^{*} \mid \mathcal{G}_{s}^{\tau}\right)=M_{t}^{*} \neq$ $M_{s}^{*}$, but it is a bounded variation $\mathbb{G}^{\tau}$-predictable process, hence a $\mathbb{G}^{\tau}$-semimartingale with null martingale part. In other terms, $\mathbb{H}$ is not immersed in $\mathbb{G}^{\tau}$ under $\mathbb{P}^{*}$.

As in Lemma 4.6, we deduce that any $(\mathbb{P}, \mathbb{G})$-martingale is a $\left(\mathbb{P}, \mathbb{G}^{\tau}\right)$-semimartingale. Note that this result can also be proved using Lemma 4.6 and a change of probability argument: a $(\mathbb{P}, \mathbb{G})$-martingale is a $\left(\mathbb{P}^{*}, \mathbb{G}\right)$ semimartingale (from Girsanov's theorem), thus also a $\left(\mathbb{P}^{*}, \mathbb{G}^{\tau}\right)$-semimartingale in view of Lemma 4.6. By another use of Girsanov's theorem, it is thus a $\left(\mathbb{P}, \mathbb{G}^{\tau}\right)$-semimartingale.

\section{Predictable Representation theorems}

The aim of this section is to obtain predictable representation property (PRP hereafter) in the enlarged filtrations $\mathbb{G}$ and $\mathbb{G}^{\tau}$, both under $\mathbb{P}$ and $\mathbb{P}^{*}$. To this end, we assume that there exists a $(\mathbb{P}, \mathbb{F})$-local martingale $z$ (possibly multidimensional), such that the PRP holds in $(\mathbb{P}, \mathbb{F})$ (cf. Assumption 5.1, below). Notice that $z$ is not necessarily continuous.

Beforehand we introduce some notation: $\mathcal{M}_{\text {loc }}(\mathbb{P}, \mathbb{F})$ denotes the set of $(\mathbb{P}, \mathbb{F})$-local martingales, and $\mathcal{M}^{2}(\mathbb{P}, \mathbb{F})$ denotes the set of $(\mathbb{P}, \mathbb{F})$-martingales $x$, such that

$$
\mathbb{E}\left(x_{t}^{2}\right)<\infty, \quad \forall t \geq 0 .
$$


Also, for a $(\mathbb{P}, \mathbb{F})$-local martingale $m$, we denote by $\mathcal{L}(m, \mathbb{P}, \mathbb{F})$ the set of $\mathbb{F}$-predictable processes which are integrable with respect to $m$ (in the sense of local martingale), namely (see, e.g., Def. 9.1 and Thm. 9.2. in He et al. [14])

$$
\mathcal{L}(m, \mathbb{P}, \mathbb{F})=\left\{\varphi \in \mathcal{P}(\mathbb{F}):\left(\int_{0} \varphi_{s}^{2} \mathrm{~d}[m]_{s}\right)^{1 / 2} \text { is } \mathbb{P} \text { - locally integrable }\right\} .
$$

Assumption $5.1(\mathrm{PRP}$ for $(\mathbb{P}, \mathbb{F}))$. There exists a process $z \in \mathcal{M}_{\mathrm{loc}}(\mathbb{P}, \mathbb{F})$ such that every $x \in \mathcal{M}_{\mathrm{loc}}(\mathbb{P}, \mathbb{F})$ can be represented as

$$
x_{t}=x_{0}+\int_{0}^{t} \varphi_{s} \mathrm{~d} z_{s}
$$

for some $\varphi \in \mathcal{L}(z, \mathbb{P}, \mathbb{F})$.

We start investigating what happens under the measure $\mathbb{P}^{*}$, in the initially enlarged filtration $\mathbb{G}^{\tau}$.

Recall that, assuming the immersion property, Kusuoka [21] has established a PRP for the progressively enlarged filtration, in the case where $\mathbb{F}$ is a Brownian filtration.

Also, under the equivalence assumption in $[0, T]$ and assuming a PRP in the reference filtration $\mathbb{F}$, Amendinger (see [1], Thm. 2.4) proved a PRP in $\left(\mathbb{P}^{*}, \mathbb{G}^{\tau}\right)$ and extended the result to $\left(\mathbb{P}, \mathbb{G}^{\tau}\right)$, in the case where the underlying (local) martingale in the reference filtration is continuous. Under the $(\mathcal{E})$-hypothesis, Grorud and Pontier [13], Proposition 4.3 have established a PRP for $\left(\mathbb{P}, \mathbb{G}^{\tau}\right)$-local martingales, in the case where the filtration $\mathbb{F}$ consists of a point process and a continuous martingale (typically a Brownian motion).

Proposition $5.2\left(\mathrm{PRP}\right.$ for $\left.\left(\mathbb{P}^{*}, \mathbb{G}^{\tau}\right)\right)$. Under Assumption 5.1, every $X^{\tau} \in \mathcal{M}_{\mathrm{loc}}\left(\mathbb{P}^{*}, \mathbb{G}^{\tau}\right)$ admits a representation

$$
X_{t}^{\tau}=X_{0}^{\tau}+\int_{0}^{t} \Phi_{s}^{\tau} \mathrm{d} z_{s}
$$

where $\Phi^{\tau} \in \mathcal{L}\left(z, \mathbb{P}^{*}, \mathbb{G}^{\tau}\right)$. In the case where $X^{\tau} \in \mathcal{M}^{2}\left(\mathbb{P}^{*}, \mathbb{G}^{\tau}\right)$, one has $\mathbb{E}^{*}\left(\int_{0}^{t}\left(\Phi_{s}^{\tau}\right)^{2} \mathrm{~d}[z]_{s}\right)<\infty$, for all $t \geq 0$ and the representation is unique.

Proof. From Theorem 13.4 in [14], it suffices to prove that any bounded martingale admits a predictable representation in terms of $z$. Let $X^{\tau} \in \mathcal{M}_{\mathrm{loc}}\left(\mathbb{P}^{*}, \mathbb{G}^{\tau}\right)$ be bounded by $K$. From Proposition 3.1, $X_{t}^{\tau}=x_{t}(\tau)$ where, for $\nu$-almost every $u \in \mathbb{R}^{+}$, the process $\left(x_{t}(u), t \geq 0\right)$ is a $\left(\mathbb{P}^{*}, \mathbb{F}\right)$-martingale, hence a $(\mathbb{P}, \mathbb{F})$-martingale. Thus Assumption 5.1 implies that (for $\nu$-almost every $u \in \mathbb{R}^{+}$),

$$
x_{t}(u)=x_{0}(u)+\int_{0}^{t} \varphi_{s}(u) \mathrm{d} z_{s}
$$

where $\left(\varphi_{t}(u), t \geq 0\right)$ is an $\mathbb{F}$-predictable process.

The process $X^{\tau}$ being bounded by $K$, it follows by an application of Lemma 2.9(i) that for $\nu$-almost every $u \geq 0$, the process $\left(x_{t}(u), t \geq 0\right)$ is bounded by $K$. Then, using the Itô isometry,

$$
\begin{aligned}
\mathbb{E}^{*}\left(\int_{0}^{t} \varphi_{s}^{2}(u) d[z]_{s}\right) & =\mathbb{E}^{*}\left(\int_{0}^{t} \varphi_{s}(u) \mathrm{d} z_{s}\right)^{2} \\
& =\mathbb{E}^{*}\left(\left(x_{t}(u)-x_{0}(u)\right)^{2}\right) \leq \mathbb{E}^{*}\left(x_{t}^{2}(u)\right) \leq K^{2} .
\end{aligned}
$$

Also, from Stricker and Yor [24], Lemma 2, one can consider a version of the process $\int_{0}^{\cdot} \varphi_{s}^{2}(u) \mathrm{d}[z]_{s}$ which is measurable with respect to $u$. Using this fact,

$$
\mathbb{E}^{*}\left[\left(\int_{0}^{t} \varphi_{s}^{2}(\tau) \mathrm{d}[z]_{s}\right)^{1 / 2}\right]=\int_{0}^{\infty} \nu(\mathrm{d} u)\left(\mathbb{E}^{*}\left(\int_{0}^{t} \varphi_{s}^{2}(u) \mathrm{d}[z]_{s}\right)\right)^{1 / 2} \leq \int_{0}^{\infty} \nu(\mathrm{d} u) K=K .
$$


The process $\Phi^{\tau}$ defined by $\Phi_{t}^{\tau}=\varphi_{t}(\tau)$ is $\mathbb{G}^{\tau}$-predictable, according to Proposition 2.7, it satisfies (5.2), with $X_{0}(\tau)=x_{0}(\tau)$, and it belongs to $\mathcal{L}\left(z, \mathbb{P}^{*}, \mathbb{G}^{\tau}\right)$.

If $X^{\tau} \in \mathcal{M}^{2}\left(\mathbb{P}^{*}, \mathbb{G}^{\tau}\right)$, from Itô's isometry,

$$
\mathbb{E}^{*}\left(\int_{0}^{t}\left(\Phi_{s}^{\tau}\right)^{2} \mathrm{~d}[z]_{s}\right)=\mathbb{E}^{*}\left(\int_{0}^{t} \Phi_{s}^{\tau} \mathrm{d} z_{s}\right)^{2}=\mathbb{E}^{*}\left(X_{t}^{\tau}-X_{0}^{\tau}\right)^{2}<\infty .
$$

Also, from this last equation, if $X^{\tau} \equiv 0$ then $\Phi^{\tau} \equiv 0$, from which the uniqueness of the representation follows.

Passing to the progressively enlarged filtration $\mathbb{G}$, which consists of two filtrations, $\mathbb{G}=\mathbb{F} \vee \mathbb{H}$, intuitively one needs two martingales to establish a PRP. Apart from $z$, intuition tells us that a candidate for the second martingale might be the compensated martingale of $H$, that was introduced, respectively under $\mathbb{P}$ (it was denoted by $M$ ) and under $\mathbb{P}^{*}$ (denoted by $M^{*}$ ), in equation (2.5) and in equation (2.6).

Proposition $5.3\left(\mathrm{PRP}\right.$ for $\left.\left(\mathbb{P}^{*}, \mathbb{G}\right)\right)$. Under Assumption 5.1, every $X \in \mathcal{M}_{\mathrm{loc}}\left(\mathbb{P}^{*}, \mathbb{G}\right)$ admits a representation

$$
X_{t}=X_{0}+\int_{0}^{t} \Phi_{s} \mathrm{~d} z_{s}+\int_{0}^{t} \Psi_{s} \mathrm{~d} M_{s}^{*}
$$

for some processes $\Phi \in \mathcal{L}\left(z, \mathbb{P}^{*}, \mathbb{G}\right)$ and $\Psi \in \mathcal{L}\left(M^{*}, \mathbb{P}^{*}, \mathbb{G}\right)$. Moreover, if $X \in \mathcal{M}^{2}\left(\mathbb{P}^{*}, \mathbb{G}\right)$, one has, for any $t \geq 0$,

$$
\mathbb{E}^{*}\left(\int_{0}^{t} \Phi_{s}^{2} \mathrm{~d}[z]_{s}\right)<\infty \quad, \quad \mathbb{E}^{*}\left(\int_{0}^{t} \Psi_{s}^{2} \lambda^{*}(s) \nu(\mathrm{d} s)\right)<\infty
$$

and the representation is unique.

Proof. It is known that any $\left(\mathbb{P}^{*}, \mathbb{H}\right)$-local martingale $\xi$ can be represented as $\xi_{t}=\xi_{0}+\int_{0}^{t} \psi_{s} \mathrm{~d} M_{s}^{*}$ for some process $\psi \in \mathcal{L}\left(M^{*}, \mathbb{P}^{*}, \mathbb{H}\right)$ (see, e.g., the proof in Chou and Meyer [7]). Notice that $\psi$ has a role only before $\tau$ and, for this reason, $\psi$ can be chosen deterministic.

Under $\mathbb{P}^{*}$, we then have:

- the PRP holds in $\mathbb{F}$ with respect to $z$;

- the PRP holds in $\mathbb{H}$ with respect to $M^{*}$;

- the filtration $\mathbb{F}$ and $\mathbb{H}$ are independent.

From classical literature (see Lem. 9.5.4.1(ii) in Jeanblanc et al. [18], for instance) the filtration $\mathbb{G}=\mathbb{F} \vee \mathbb{H}$ enjoys the PRP under $\mathbb{P}^{*}$ with respect to the pair $\left(z, M^{*}\right)$.

Now suppose that $X \in \mathcal{M}^{2}\left(\mathbb{P}^{*}, \mathbb{G}\right)$. We find

$$
\begin{aligned}
\infty & >\mathbb{E}^{*}\left(X_{t}-X_{0}\right)^{2}=\mathbb{E}^{*}\left(\int_{0}^{t} \Phi_{s} \mathrm{~d} z_{s}+\int_{0}^{t} \Psi_{s} \mathrm{~d} M_{s}^{*}\right)^{2} \\
& =\mathbb{E}^{*}\left(\int_{0}^{t} \Phi_{s}^{2} \mathrm{~d}[z]_{s}\right)+2 \mathbb{E}^{*}\left(\int_{0}^{t} \Phi_{s} \mathrm{~d} z_{s} \int_{0}^{t} \Psi_{s} \mathrm{~d} M_{s}^{*}\right)+\mathbb{E}^{*}\left(\int_{0}^{t} \Psi_{s}^{2} \lambda^{*}(s) \nu(\mathrm{d} s)\right),
\end{aligned}
$$

where in the last equality we used the Itô isometry. The cross-product term in the last equality is zero due to the orthogonality of $z$ and $M^{*}$ (under $\mathbb{P}^{*}$ ). From this inequality, the desired integrability conditions hold and the uniqueness of the representation follows (as in the previous proposition).

Remark 5.4. In order to establish a PRP for the initially enlarged filtration $\mathbb{G}^{\tau}$ and under $\mathbb{P}^{*}$, one could have proceeded as in the proof of Proposition 5.3, noting that any martingale $\xi$ in the "constant" filtration $\sigma(\tau)$ satisfies $\xi_{t}=\xi_{0}+0$ and that under $\mathbb{P}^{*}$ the two filtrations $\mathbb{F}$ and $\sigma(\tau)$ are independent. 
Proposition 5.5 (PRP under $\mathbb{P})$. Under Assumption 5.1, one has:

(i) every $X^{\tau} \in \mathcal{M}_{\mathrm{loc}}\left(\mathbb{P}, \mathbb{G}^{\tau}\right)$ can be represented as

$$
X_{t}^{\tau}=X_{0}^{\tau}+\int_{0}^{t} \Phi_{s}^{\tau} \mathrm{d} Z_{s}^{\tau}
$$

where $Z^{\tau}$ is the martingale part in the $\mathbb{G}^{\tau}$-canonical decomposition of $z$ and $\Phi \in \mathcal{L}\left(Z^{\tau}, \mathbb{P}, \mathbb{G}^{\tau}\right)$;

(ii) every $X \in \mathcal{M}_{\mathrm{loc}}(\mathbb{P}, \mathbb{G})$ can be represented as

$$
X_{t}=X_{0}+\int_{0}^{t} \Phi_{s} \mathrm{~d} Z_{s}+\int_{0}^{t} \Psi_{s} \mathrm{~d} M_{s}
$$

where $Z$ is the martingale part in the $\mathbb{G}$-canonical decomposition of $z$ (cf. Eq. (4.3)), $M$ is the $(\mathbb{P}, \mathbb{G}$ )compensated martingale associated with $H$ and $\Phi \in \mathcal{L}(Z, \mathbb{P}, \mathbb{G}), \Psi \in \mathcal{L}(M, \mathbb{P}, \mathbb{G})$.

Proof. The assertion (i) (resp. (ii)) follows from Proposition 5.2 (resp. Prop. 5.3) and the stability of PRP under an equivalent change of measure (see for example Thm. 13.12 in [14]).

The PRP for the progressively enlarged filtration with a random time (i.e., part (ii) of the above proposition), has been first presented by Jeanblanc and Le Cam [17]. Our proof has the advantage that it can be straightforwardly generalized to the case where $\tau$ is a vector of random times, as is discussed in the last section.

\section{Concluding Remarks}

We conclude the paper with some important comments:

- In the multidimensional case, that is when $\tau=\left(\tau_{1}, \ldots, \tau_{d}\right)$ is a vector of finite random times, the same machinery can be applied. More precisely, under the assumption

$$
\mathbb{P}\left(\tau_{1} \in \mathrm{d} \theta_{1}, \ldots, \tau_{d} \in \mathrm{d} \theta_{d} \mid \mathcal{F}_{t}\right) \sim \mathbb{P}\left(\tau_{1} \in \mathrm{d} \theta_{1}, \ldots, \tau_{d} \in \mathrm{d} \theta_{d}\right)
$$

one defines the probability $\mathbb{P}^{*}$ equivalent to $\mathbb{P}$ on $\mathcal{G}_{t}^{\tau}=\mathcal{F}_{t} \vee \sigma\left(\tau_{1}\right) \vee \cdots \vee \sigma\left(\tau_{d}\right)$ by

$$
\left.\frac{\mathrm{d} \mathbb{P}^{*}}{\mathrm{dP}}\right|_{\mathcal{G}_{t}^{\tau}}=\frac{1}{p_{t}\left(\tau_{1}, \ldots, \tau_{d}\right)},
$$

where $p_{t}\left(\tau_{1}, \ldots, \tau_{d}\right)$ is the (multidimensional) analog to $p_{t}(\tau)$, and the results for the initially enlarged filtration are obtained in the same way as for the one-dimensional case.

As for the progressively enlarged filtration, we define $\mathbb{H}:=\mathbb{H}^{1} \vee \cdots \vee \mathbb{H}^{d}$, where $\mathbb{H}^{i}$ stands for the natural filtration of the indicator process $H^{i}=\left(\mathbb{1}_{\tau_{i} \leq t}, t \geq 0\right)$. The progressive enlargement of $\mathbb{F}$ with the vector $\left(\tau_{1}, \ldots, \tau_{d}\right)$, is then defined by (the right-continuous regularization of) the filtration $\mathbb{F} \vee \mathbb{H}$. One has to note that, in this case, a measurable process is decomposed into $2^{d}$ terms, corresponding to the measurability of the process on the various sets $\left\{\tau_{i} \leq t<\tau_{j}, i \in I, j \in I^{c}\right\}$ for all the subsets $I$ of $\{1, \ldots, d\}$.

An interesting point is the generalization of the proof of predictable representation theorem for the progressively enlarged filtration, in the multidimensional case. Under the probability $\mathbb{P}^{*}$, defined in (6.1), the filtration $\mathbb{H}$ is independent of $\mathbb{F}$. So, once a PRP for $\mathbb{H}$ holds true, it is straightforward to generalize Proposition 5.3 to the multidimensional case. To this end, we further assume that the (joint) law $\mathbb{P}\left(\tau_{1} \in \mathrm{d} \theta_{1}, \ldots, \tau_{d} \in \mathrm{d} \theta_{d}\right)$ of $\tau$ is absolutely continuous w.r.t. the Lebesgue measure on $\left(\mathbb{R}^{+}\right)^{d}$. One then has $\mathbb{P}\left(\tau_{i}=\tau_{j}\right)=0$ and thus 
the process $H=\left(H^{1}, \ldots, H^{n}\right)$ is an $n$-variate point process (in the terminology of Brémaud [6]). So, $\mathbb{H}$ enjoys the PRP with respect to the compensated martingales of $H^{1}, \ldots, H^{n}$ (see for instance Brémaud [6] Chap. III, Sect. 3, Thms. 9 and 11);

- in this study, honest times (recall that a random time $L$ is honest if, for any $t$, it is equal to an $\mathcal{F}_{t}$-measurable random variable on $\{L<t\}$ ) are automatically excluded, as we explain now. Under the probability $\mathbb{P}^{*}$, the Azéma supermartingale associated with $\tau$, being a continuous decreasing function, has a trivial Doob-Meyer decomposition $G^{*}=1-A^{*}$ with $A_{t}^{*}=\int_{0}^{t} \nu(\mathrm{d} u)$. So, $A_{\infty}^{*}=1$ and, in particular, $\tau$ can not be an honest time: recall that in our setting, $\tau$ avoids the $\mathbb{F}$-stopping times and therefore, from a result due to Azéma (cf. [3], part (b) of the Thm. in pp. 300 and 301), if $\tau$ is an honest time, the random variable $A_{\infty}^{*}$ should have exponential law with parameter 1, which is not the case (note that the notion of honest time does not depend on the probability measure);

- under the $(\mathcal{E})$-hypothesis, the immersion property between $\mathbb{F}$ and $\mathbb{G}$ is equivalent to $p_{t}(u)=p_{u}(u), t \geq u$ ( $c f$. [16], Cor. 3.1). In particular, as expected, the canonical decomposition formula presented in Proposition 4.3 is trivial, that is, the two integral terms on the right-hand side of (4.3) vanish;

- Predictable representation theorems can be obtained in the more general case, where any $(\mathbb{P}, \mathbb{F})$-martingale $x$ admits a representation as

$$
x_{t}=x_{0}+\int_{0}^{t} \int_{E} \varphi(s, \theta) \widetilde{\mu}(\mathrm{d} s, \mathrm{~d} \theta)
$$

for a compensated martingale associated with a point process.

Acknowledgements. The authors would like to thank Prof. Sh. Song (UEVE, France) and Prof. M. Pontier (Paul Sabatier, Toulouse, France) for their enlightening discussions and precious remarks.

\section{REFERENCES}

[1] J. Amendinger, Initial Enlargement of Filtrations and Additional Information in Financial Markets. Ph.D. thesis, Technischen Universität Berlin (1999).

[2] S. Ankirchner, S. Dereich and P. Imkeller, Elargement of filtrations, continuous Girsanov-type embeddings, Séminaire de probabilités XL (2007) 389-410.

[3] J. Azéma, Quelques applications de la théorie générale des processus, Invent. Math. 18 (1972). $293-336$.

[4] M.T. Barlow, Study of filtration expanded to include an honest time. Z. Wahr. Verw. Gebiete 44 (1978) $307-323$.

[5] T.R. Bielecki, M. Jeanblanc and M. Rutkowski, Credit Risk Modeling. CSFI Lect. Note Series. Osaka University Press (2009).

[6] P. Brémaud, Point Processes and Queues: Martingale Dynamics. Springer-Verlag (1981).

[7] C.S. Chou and P.-A. Meyer, Sur la représentation des martingales comme intégrales stochastiques dans les processus ponctuels. Séminaire de probabilités IX (1975) 226-236.

[8] C. Dellacherie and P.-A. Meyer, Probabilités et Potentiel - Chapitres XXVII à XXIV, Processus de Markov. Hermann, Paris (1992).

[9] N. El Karoui, M. Jeanblanc and Y. Jiao, What happens after a default: the conditional density approach. Stoch. Proc. Appl. 120 (2010) 1011-1032.

[10] H. Föllmer and P. Imkeller, Anticipation cancelled by a Girsanov transformation: a paradox on Wiener space. Ann. Inst. Henri Poincaré 29 (1993) 569-586.

[11] D. Gasbarra, E. Valkeila and L. Vostrikova, Enlargement of filtration and additional information in pricing models: Bayesian approach, in From Stochastic Calculus to Mathematical Finance, edited by Y. Kabanov, R. Liptser and J. Stoyanov. SpringerVerlag (2006) 257-285.

[12] A. Grorud and M. Pontier, Insider trading in a continuous time market model. Int. J. Theor. Appl. Finance 1 (1998) 331-347.

[13] A. Grorud and M. Pontier, Asymmetrical information and incomplete markets. Int. J. Theor. Appl. Finance 4 (2001) $285-302$.

[14] Sh. He, J. Wang and J. Yan, Semimartingale theory and stochastic calculus. CRC Press (1992).

[15] J. Jacod, Grossissement initial, hypothèse $\left(\mathrm{H}^{\prime}\right)$ et théorème de Girsanov, Lect. Notes Math., vol. 1118. Springer-Verlag (1985) $15-35$.

[16] M. Jeanblanc and Y. Le Cam, Progressive enlargement of filtrations with initial times. Stoch. Proc. Appl. 119 (2009) $2523-2543$. 
[17] M. Jeanblanc and Y. Le Cam, Immersion Property and Credit Risk Modelling, in Optimality and Risk - Modern Trends in Mathematical Finance, edited by F. Delbaen, M. Rásonyi and C. Stricker. Springer (2010) 99-132.

[18] M. Jeanblanc, M. Yor and M. Chesney, Mathematical Methods in Financial Markets. Springer (2009).

[19] T. Jeulin, Semimartingales et grossissement d'une filtration, Lect. Notes Math., vol. 833. Springer-Verlag (1980).

[20] Y. Kchia, M. Larsson and P. Protter, Linking progressive and initial filtration expansions, Working paper.

[21] S. Kusuoka, A remark on default risk models, Adv. Math. Econ. 1 (1999) 69-82.

[22] Sh. Song, Grossissement de filtration et problèmes connexes. Ph.D. thesis, Université Paris VI (1987).

[23] C. Stricker, Quasi-martingales, martingales locales et filtrations naturelles. Zeitschrift fur Wahr 39 (1977) 55-63.

[24] C. Stricker and M. Yor, Calcul stochastique dépendant d'un paramètre. Zeitschrift fur Wahr 45 (1978) 109-133.

[25] M. Yor, Grossissement de filtrations et absolue continuité de noyaux, Lect. Notes Math., vol. 1118. Springer-Verlag (1985) $7-14$. 